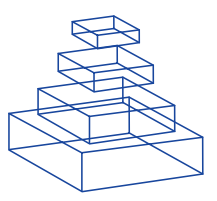

\title{
The neuroanatomist's dream, the problems and solutions, and the ultimate aim
}

\author{
Javier DeFelipe \\ Cajal Institute (CSIC), Spain \\ Correspondence: defelipe@cajal.csic.es
}

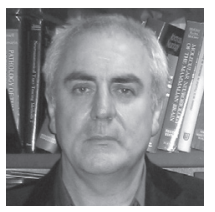

Javier DeFelipe is Professor of Neuroanatomy at the Cajal Institute (CSIC).

He was a PhD student at

the Universidad Complutense (Madrid) in 1979 under the supervision of Dr. José Rodrigo at the Cajal Institute. After postdoctoral training (1980-1983) at the the Cajal Institute with Dr. Alfonso

Fairén and Dr. Facundo Valverde, he obtained a Fogarty Fellowship (NIH) to work with Dr. Edward Jones at the Washington University School of Medicine, St. Louis (USA). In 1986, DeFelipe obtained tenure in Neuroscience at the Cajal Institute. In 2000, he was appointed as Research Scientist, and in 2004 as Full Professor in the same institution. His research focuses on the microorganization of the normal cerebral cortex (including hippocampus) in various species and on the alterations of cortical circuits in epilepsy and Alzheimer disease.
The principal goal in neuroanatomy is to define the detailed structural design of the nervous system. This challenge is one of the first steps towards understanding how neural circuits contribute to the functional organization of the nervous system, both in health and disease. The main difficulties involve unraveling the extraordinary complexity of the nervous system and to define how information flows through this finely organized synaptic network. Over the years, neuroanatomy has evolved considerably thanks to the use of classical techniques and the introduction of new procedures (DeFelipe, 2002), including: axonal transport methods to trace connections; electron microscopy to better understand synaptic connectivity; immunocytochemistry to map protein expression and the distribution of particular types of neurons; in situ hybridization to map gene expression; intracellular labeling of physiologically characterized neurons to visualize their morphology; and other powerful techniques to examine the organization of the nervous system. Among the most appealing new tools are the molecular/genetic approaches to activate, inactivate and label active neurons and synapses (Marek and Davis, 2003; Dymecki and Kim, 2007; Kuhlman and Huang, 2008). In turn, as more detailed circuit diagrams become available, the more we will learn about the role of each element in the circuit through computer simulations, and this will enable the response properties of individual neurons to be correlated with the activity in cortical circuits, an attractive bridge between anatomy, physiology and computation (Segev and London, 2000; Markram, 2006). Additionally, the magnitude of morphological, molecular and physiological data collectively generated is now so colossal that even at the cellular level it is necessary to develop neuronal classification systems, in order to help the scientific community in each field to establish and maintain an effective communication and interchange of information (Ascoli et al., 2008). Thus, we have to learn not only how to manage the growing amount of multidisciplinary data but also, how to communicate this knowledge.

\section{The dream}

The neuroanatomist's dream is to characterize at the light microscope level the detailed neurochemical and 3D morphology of the neurons and glial cells in the brain, and of the relationships between them (neuron/neuron; neuron/glia; glia/glia), as well as to create a 
3D map of the synaptic network in the entire nervous system at the electron microscope level. Ideally, this detailed morphological information should be correlated with other features such as the molecular and physiological characteristics of the cells under study. However, is it really important to generate 3D reconstructions of neurons and circuits? At the light microscope level, the pattern of axonal arborization is generally the main determinant of a neurons' output connectivity. By contrast, the morphology of the dendritic tree is related to the processing of inputs. For example, the structure of the dendritic tree itself affects the process of integration, while its size influences topographic sampling strategies and the mixing of inputs (Koch and Segev, 2000). Electron microscopy reconstructions, in contrast to single section analysis, are the best way to quantitatively analyze key ultrastructural characteristics of axonal and dendritic processes (e.g., density and type of synaptic vesicles, density of mitochondria), synaptic connectivity (e.g., types of synapses, identification of synaptic targets), the relationship between glial processes and synapses, etc. Therefore, visualizing and analyzing the complete dendritic and axonal arbors of neurons and glial processes, and their 3D ultrasturural characteristics and synaptic connections, is tremendously important in terms of function.

\section{The problems}

The above analyses are still subject to several major limitations. There are two main obstacles at the light microscope level. First, it is necessary to use relatively thin tissue slices to visualize labeled neurons, frequently in the order of a few microns, in contrast to the hundreds of microns or even millimeters over which neuronal processes may expand. Currently, this problem can only be overcome by using serial sections to reconstruct the cell in 3D. The second major limitation is that the processes are not always easy to trace and they may be lost in the background noise at times. Together, these drawbacks make it very laborious and time-consuming to obtain meaningful measurements from neurons and thus, automated techniques must be developed to assist in the reconstruction of axons and dendrites (Schmitt et al., 2004; Macke et al., 2008). There are also two important problems at the electron microscope level. First, obtaining long series of ultrathin sections is extremely time-consuming and difficult, often making it impossible to completely reconstruct neurons or their processes (particularly axons). This makes electron microscopy impractical to study large numbers of individual neurons in detail, or to reconstruct large volumes of tissue. Therefore, most studies are based on the analysis of a few cells, limiting the value of the general conclusions drawn from these observations. Hence, the development of automated electron microscopy techniques will represent another important step in the study of neuronal circuits (e.g., serial section scanning electron microscopy using focused ion beam milling: Knott et al., 2008). A second problem that is often disregarded is the difficulty in studying synaptic inputs to labelled neurons. Most techniques used to visualize neurons produce intense, homogeneous intracellular staining, which unfortunately mask post-synaptic densities (such as the deposit produced by the chromogen 3,3' diaminobenzidine tetrahydrochloride). This makes it difficult or in many cases impossible to identify synaptic inputs when the presynaptic element is also labelled. This is what I call in laboratory jargon "arrowed synapses", those that are only seen when indicated by an arrow in a figure. Thus, this problem represents another important challenge and techniques to label neurons without masking their ultrastructure must be used (e.g., techniques that employ gold particles).

Furthermore, full reconstruction of whole brains at the electron microscope level is possible in some invertebrates, or for relatively simple nervous systems. However, even for a small mammal like the mouse it is impossible to fully reconstruct the brain at this level. The magnification needed to visualize chemical synapses yield microscopy images of at least $10 \times 10$ microns in size (higher magnification is needed in the case of electrical synapses, making these fields even smaller), so to reconstruct only $1 \mathrm{~mm}^{3}$ would require the assembly of a stack of around $10^{9}$ images. Full reconstructions of a small region of the mammalian brain are feasible, but structures like the cerebral cortex which can reach a surface area of $2,200 \mathrm{~cm}^{2}$ and a thickness that varies between 1.5 to $4.5 \mathrm{~mm}$ in humans is certainly not possible. 


\section{REFERENCES}

Ascoli, G. A., Alonso-Nanclares, L., Anderson, S. A., Barrionuevo, G., Benavides-Piccione, R. (2008). Petilla Terminology: Nomenclature of features of GABAergic interneurons of the cerebral cortex. Nat. Rev. Neurosci. 9, 557-568.

DeFelipe, J. (2002). Cortical interneurons: From Cajal to 2001. Prog. Brain Res. 136, 215-238.

DeFelipe, J., Alonso-Nanclares, L., Arellano, J., Ballesteros-Yáñez, I., Benavides-Piccione, R., Munoz, A. (2007). Specializations of the cortical microstructure of humans. In: Evolution of the nervous systems: a comprehensive reference. Vol. 4. Primates. Kaas, J. H. \& Preuss, T. M. (eds.). Amsterdam, Elsevier, 2007, 167-190.

Dymecki, S. M., Kim, J. C. (2007). Molecular neuroanatomy's "Three Gs": a primer. Neuron. 54, 17-34.

\section{The solutions}

Thus, what can we do? First, it is important to bear in mind that although synaptic circuits within a given area and layer vary, this variability remains within a relatively narrow window. For example, in the rat hindlimb somatosensory cortex, the range of all synaptic profiles per $100 \mu \mathrm{m} 2$ of neuropil only varies between 32 and 46 . Similarly, it is expected that the variation among the ultrastructural characteristics will also fall within narrow windows. Therefore, we do not need to reconstruct the whole layer within a given area to find out the absolute number and types of synapses, and to study the ultrastructural characteristics, but rather the range of variability can be ascertained by multiple sampling of relatively small areas within that region. Thus, the strategy to unravel the complex organization of the brain is to combine light microscopy information with 3D electron microscopy. In addition, enterprises like the Blue Brain Project (Markram, 2006) will be necessary to develop tools to manage the thousands of terabytes of data that this kind of analysis will generate. This will enable us to store, retrieve and analyze these huge databases of information on morphology, molecular features and connectivity in order to try to correlate the $3 \mathrm{D}$ microanatomy of a given region with its functional activity, in order words, to try to unravel the microanatomical substrate of brain activity.

\section{The ultimate aim}

Finally, it is also important to remember that the ultimate aim of neuroscience is to understand the human brain. Although many structural aspects of the nervous system are phylogenetically conserved, others are unique to humans (DeFelipe et al., 2007). Therefore, we have to face the additional ethical problems associated with the application of certain anatomical techniques to human material. As a result, there are considerable voids in the information available that is directly related to human brain microanantomy. Accordingly, any advances in this direction would be welcomed, like the development of brain imaging techniques (e.g., "diffusion tensor" magnetic resonance imaging that provides amazing images of tracts of nerve fibers in vivo) or methods to perform intracellular injections in fixed material that facilitate the use of autopsy material. To conclude, the technical advances achieved in recent years and the new technologies that are under development make neuroanatomy one of most exciting and important fields in neuroscience.

Koch, C., Segev, I. (2000). The role of single neurons in information processing. Nat. Neurosci. 3, 1171-1177.

Knott, G., Marchman, H., Wall, D., Lich, B. (2008). Serial section scanning electron microscopy of adult brain tissue using focused ion beam milling. J. Neurosci. 28, 2959-2964.

Kuhlman, S. J., Huang, Z. J. (2008). High-resolution labeling and functional manipulation of specific neuron types in mouse brain by Cre-activated viral gene expression. PLoS ONE Apr 16; 3:4, e2005.

Macke, J. H., Maack, N., Gupta, R., Denk, W., Schölkopf, B., Borst, A. (2008). Contour-propagation algorithms for semi-automated reconstruction of neural processes. J. Neurosci. Methods. $167: 2,349-357$.

Markram, H. (2006). The blue brain project. Nat. Rev. Neurosci. 7, 153-160.
Marek, K. W., Davis, G. W. (2003). Controlling the active properties of excitable cells. Curr. Opin. Neurobiol. 13, 607-611.

Schmitt, S., Evers, J. F., Duch, C., Scholz, M., Obermayer, K. (2004). New methods for the computer-assisted 3 -D reconstruction of neurons from confocal image stacks. Neuroimage. 23, 1283-1298.

Segev, I., (2000). Untangling Dendrites with Quantitative Models. Science. 290, 744-750.

Front. Neurosci. (2008) 2, 1: 10-12. doi: 10.3389/ neuro.01.018.2008

Copyright (C) 2008 DeFelipe. This is an openaccess publication subject to an exclusive license agreement between the authors and the Frontiers Research Foundation, which permits unrestricted use, distribution, and reproduction in any medium, provided the original authors and source are credited. 